Stefan Kubica, Hagen Ringshausen,

Jörg Reiff-Stephan, Marius Schlingelhof (Hrsg.)

2. Automobil Symposium Wildau:

Tagungsband Technische Hochschule Wildau 2017
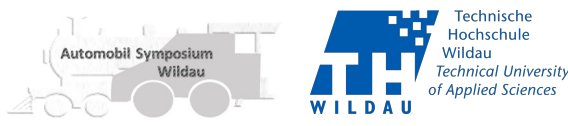

\author{
Mitko Dimitrow \\ RevDop GmbH \\ mitko.dimitron@revdop.de
}

\author{
Robin Böttcher \\ RevDop GmbH \\ robin.boettcher@revdop.de
}

\author{
Matthias Bierig \\ RevDop GmbH \\ matthias.bierig@revdop.de
}

\title{
REENGINEERING IM RAPID-PROTOTYPING
}

\section{Zusammenfassung}

Die RevDop GmbH wurde am 01.12.2016 als $\mathrm{GmbH}$ (i. G.) gegründet, um innovative Strömungsbauteile für Verbrennungsmotoren, mit dem Ziel, den Wirkungsgrad der Motoren zu steigern, zu entwickeln. Für dieses Projekt erhält das RevDop-Team seit Dezember 2016 das Exist-Gründerstipendium. Ein Förderprogramm, welches vor allem wissenschaftsbasierende Gründungsvorhaben mit einem hohen Grad an Innovation unterstützen soll. Neben der eigenen Produktentwicklung befindet sich die RevDop $\mathrm{GmbH}$ aktuell in einer Phase des Know-How-Aufbaus für moderne Reverse Engineering Prozesse, welche in Form eines Serviceportfolios für die Produktentwicklung vorrangig im Bereich Automotive Engineering angeboten werden. In den Kapiteln 2 und 3 werden beide Themenbereiche beschrieben.

\section{Einführung}

Zunächst werden die beiden Kernkompetenzen des RevDop-Teams im Überblick dargestellt.

\subsection{Entwicklung innovativer Strömungsbauteile für Verbrennungsmotoren}

Aufgrund von Verlustprozessen erreichen moderne Verbrennungsmotoren auch heute noch Wirkungsgrade von lediglich 40 bis $50 \%$. Erheblichen negativen Einfluss haben dabei Ladungswechselverluste im Ansaug- und Abgasbereich, die hauptsächlich auf die Verwendung von Standardgeometrien bei der Entwicklung von Strömungsbauteilen zurückzuführen sind. Die RevDop GmbH entwickelt innovative Strömungsbauteile für Dieselund Benzinmotoren. Durch die Realisierung von Strömungsgeometrien, die sich an den physikalischen Gegebenheiten der Natur orientieren, wird der Druckverlust innerhalb der Ansaug- und Abgassysteme um bis zu $60 \%$ minimiert. Dadurch kann der Wirkungsgrad der Motoren erheblich gesteigert werden. In einem Pilotprojekt wurde mit der Optimierung eines Teilsystems im Ansaugbereich eines PKW eine Wirkungsgradsteigerung um circa $6 \%$ erreicht. Durch die Steigerung des Wirk- 
ungsgrades werden der Kraftstoffverbrauch und der Schadstoffausstoß reduziert und die Leistungsabgabe erhöht. Bei der Entwicklung dieser Produkte ist ein hohes Maß an Know-How in verschiedenen Ingenieurprozessen erforderlich. Die Erfahrung in der Produktentwicklung von Automobilbauteilen nutzt das RevDop-Team und bietet einen effizienten und qualitätsbewussten Produktentwicklungsprozess als Service an.

\subsection{Re-Engineering-Service als Output der Produktentwicklung}

Im Rahmen der Entwicklung und Fertigung von Automobilbauteilen kommt es verstärkt zur Forderung nach individuell angepassten Lösungen. RevDop bedient sich deshalb modernster Verfahren, um individuelle Anforderungen in kurzen Entwicklungsphasen zu realisieren. Durch den Einsatz moderner Generativen Fertigungsverfahren und zukünftig auch mithilfe von Virtual Reality, ist es möglich bereits in sehr frühen Entwicklungsstadien realistische Produktpräsentationen und Funktionstests durchzuführen. Dadurch können kostenintensive Fehler vermieden und frühzeitig Konzeptänderungen und Optimier-

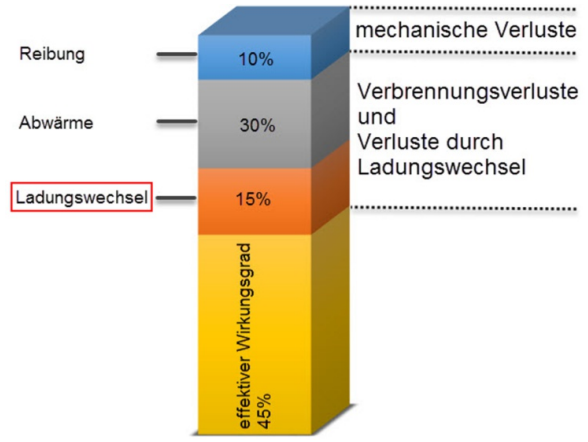

ungen durchgeführt werden. Die Entwicklungsphasen von der Produktidee bis zum Serienbauteil können so deutlich verkürzt werden.

\section{RevDop - Naturorientierte Strömungsbauteile}

\subsection{Einleitung, Problemdarstellung}

Der Wirkungsgrad moderner Benzinund Dieselmotoren liegt zwischen 40 und 50 Prozent. Durch Verlustprozesse geht ein Großteil des Kraftstoffes verloren (50 bis 60 Prozent) und wird nicht für die Fortbewegung des Fahrzeuges genutzt. Einen dieser Verlustprozesse stellt der Ladungswechsel in Ansaugund Abgassystemen dar, der den Wirkungsgrad um bis zu 15 Prozent negativ beeinflusst (Abbildung 1). Dadurch steigt der Kraftstoffverbrauch deutlich (Bei Betrachtung des gesamten Strömungssystems, je nach Motortyp um bis $\mathrm{zu} 25$ Prozent). Dies hat einen höheren Schadstoffausstoß zur Folge. Ebenfalls wird die Leistung erheblich beeinträchtigt. Um dies zu vermeiden ist es entscheidend Strömungsverluste in diesen Systemen zu minimieren.

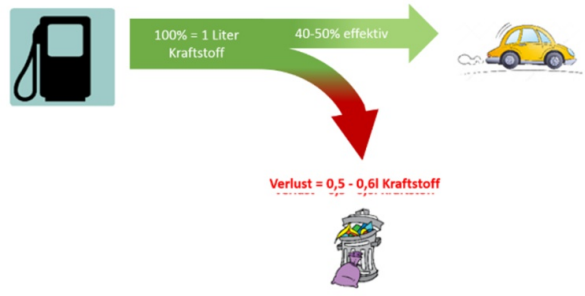


Abbildung 2 stellt den spezifischen Kraftstoffverbrauch im Verhältnis zum Druckverlust in Abgassystemen dar. Es ist deutlich zu erkennen, dass der spezifische Brennstoffverbrauch mit $\mathrm{Zu}$ nahme des Gegendruckes, besonders im
Feinstaubbelastung in Städten. Darüber hinaus wird die Leistungsfähigkeit des Motors erheblich beeinträchtigt. [Zell12] [KuBe09] [Simo15]

Die standardmäßig aber auch bereits verbesserten Ansaug- und Abgassysteme

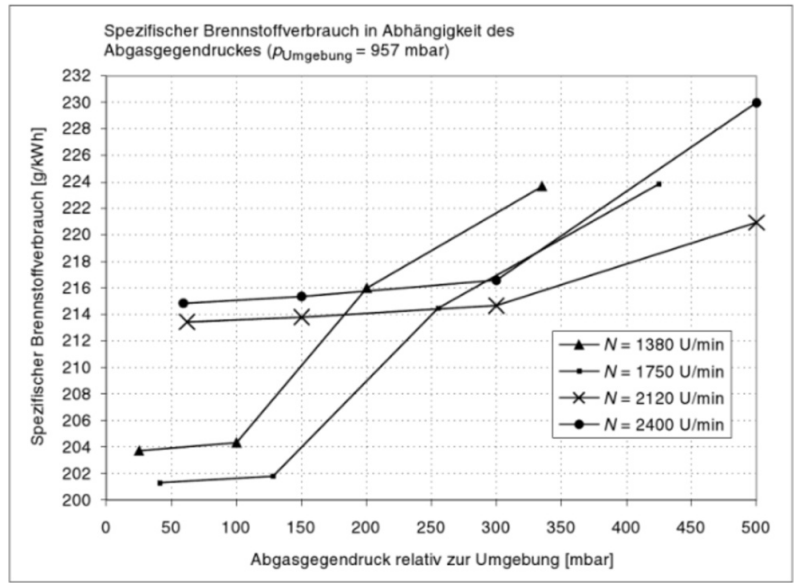

Abbildung 2: Kraftstoffverbrauch in Abhängigkeit zum Druckverlust in Abgassystemen [BaSc15]

niedrigen Drehzahlbereich, wie er häufig im Stadtverkehr Anwendung findet, ansteigt. Dies kann näherungsweise auch auf Ansaugsysteme übertragen werden, da diese, wie auch Abgassysteme, zum Vorgang des Ladungswechsels gehören. Der erhöhte Kraftstoffverbrauch hat einen gesteigerten Schadstoffausstoß zur Folge. Dadurch ergibt sich eine erhöhte haben erhebliche Schwachstellen, die die Strömungsführung negativ beeinflussen. Nahezu alle Strömungssysteme werden mit abrupten Querschnittsänderungen, Standard- $90^{\circ}$-Rohrbögen, strömungshindernden Steckverbindungen und Standardgeometrien realisiert, bei denen enorme Druckverluste entstehen.

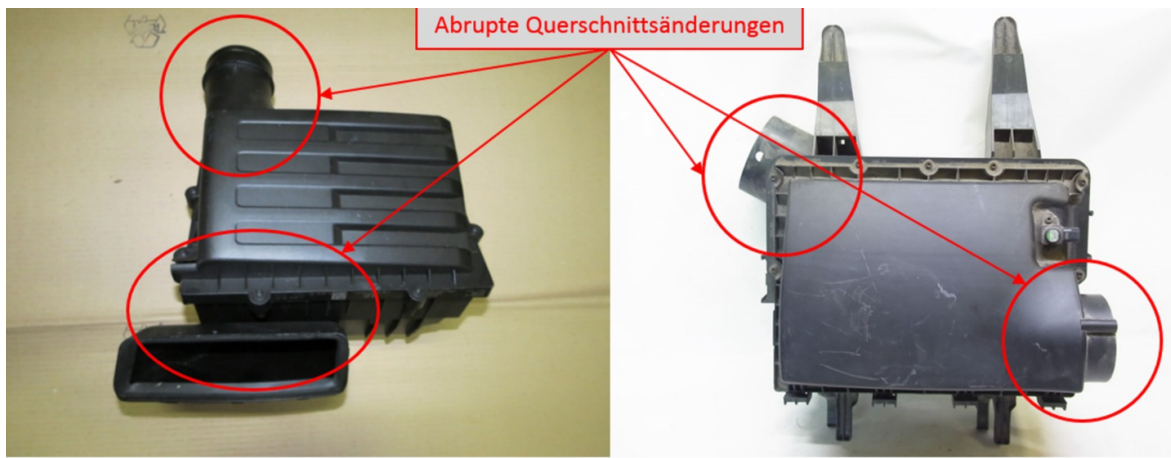

Abbildung 3: abrupte Querschnittsänderungen Golf 7 R (links), Mercedes Sprinter (rechts) 




Abbildung 4: Anwendungsfall $90^{\circ}$ Rohrbogen (links) [eigene Darstellung], Schwachstellen Audi Q3

[Hohe15]

Abbildung 3 zeigt Beispiele von mittels Standardgeometrien realisierter Luftfilterkästen mit abrupten Querschnittsänderungen eines Mercedes Sprinter (rechts) und eines Golf 7 R (links). Dies betrifft Ansaug- und Abgassysteme gleichermaßen.Zudem werben zwar einige Tuningunternehmen mit einer Leistungssteigerung durch ihre Produkte, jedoch werden diese nur in sehr seltenen Fällen mit offiziellen Prüfprotokollen belegt. Ein demonstratives Beispiel für ein standardmäßig verbautes, suboptimales Ansaugsystem des Automobilherstellers Audi wird in Abbildung 4 dargestellt.

Abbildung 5 zeigt Schwachstellen (rot eingekreist) bereits optimierter Strömungssysteme etablierter Tuningunternehmen. Optimierte Systeme mit vergleichbaren Schwachstellen lassen sich bei nahezu allen Produkten von Unternehmen, welche verbesserte Strömungssysteme für PKW-Motoren entwickeln, finden. Im Bereich der Nutzkraftfahrzeuge gibt es derzeit keinen einzigen Anbieter, der Strömungsbauteile von Verbrennungsmotoren optimiert. [Müll01, pp. 27ff,] [BüKa12, p. 70f.] [11] Automobilhersteller sind aufgrund Ihrer Arbeitsphilosophie, Ihrer Vernetzung mit Zulieferunternehmen, ihrer komplexen Produktionsprozesse und aus wirtschaftlichen Gründen bisher an das Regelwerk der parametrischen Konstruktion gebunden, was eine Umsetzung optimaler Strömungssysteme unmöglich macht. [8, pp. 3ff., 156ff., 172ff., 199ff.] [3] [4] [Müll01] [10, p. 90ff.]

\subsection{Einleitung, Problemdarstellung}

Um alle Schwachstellen bestehender Strömungssysteme nahezu eliminieren zu können, hat RevDop eigene Optimierungs-Techniken entwickelt. Dabei werden alle negativen Einflussfaktoren, wie ungünstige Anströmwinkel von Luftfiltern, eckige Luftfilterformen, abrupte Querschnittsänderungen, eckige Querschnittsformen und druckverluststeigernde Strömungsumlenkungen 


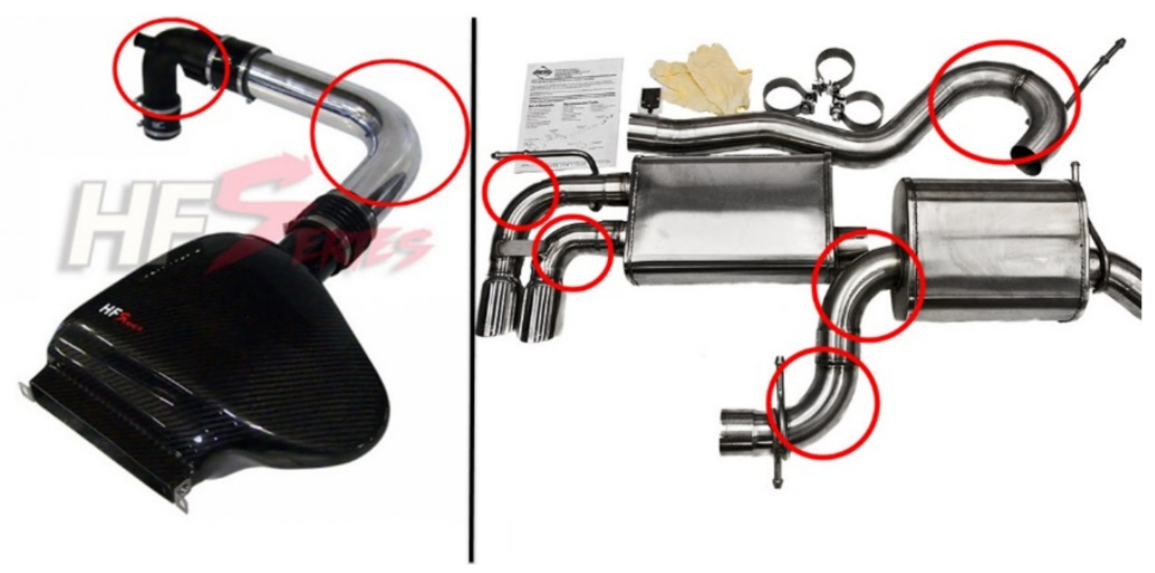

Abbildung 5: Ansaugsystem HG-Motorsport (links) [Gükd12], Auspuffanlage APR (rechts) [Ston12]

durch Rohrbögen in bestehenden Systemen analysiert und, unter Berücksichtigung der physikalischen Gegebenheiten der Natur, optimiert. Mithilfe der Topologieoptimierung und einer parameterfreien Konstruktionsweise werden naturorientierte Strömungsgeometrien realisiert. Dadurch wird der maximal mögliche Optimierungsgrad in Ansaugund Abgassystemen erreicht. Der stratives Beispiel ist ein $180^{\circ}$ Flusslauf, deren charakteristische Form auf einen Rohrbogen übertragen werden kann, Abbildung 6 .

Um optimale, kompromisslose Ansaugsysteme umsetzen zu können, hat RevDop als erstes Unternehmen eigene Optimierungstechniken entwickelt, die in ihrer Kombination herausragende Innovationen darstellen. Diese werden in
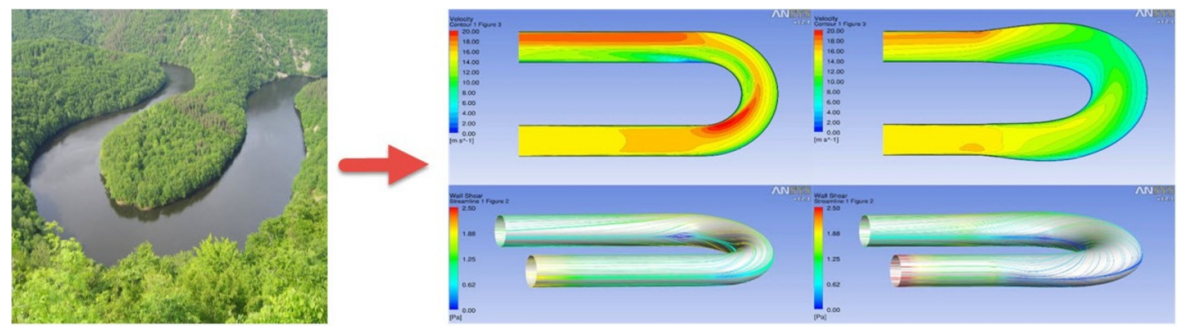

Abbildung 6: Abbildung 6: Übertragung Flusslauf auf Rohrbogen [BüKa12, p. 71]

Druckverlust in Ansaug- und Abgassystemen wird um bis zu 60 Prozent gesenkt. Dadurch wird der Wirkungsgrad um bis zu 15 Prozent gesteigert, was den Kraftstoffverbrauch und den Schadstoffausstoß erheblich senkt. Ein demon- den Abschnitten 2.2.1 bis 2.2.7 beschrieben. RevDop vereint als erstes Unternehmen die physikalischen Gegebenheiten der Natur mit selbstentwickelten Techniken zur Umsetzung der wissenschaftlichen Strömungslehre 
und schafft damit Strömungssysteme, welche aufgrund einer nahezu optimalen Strömungsführung den Wirkungsgrad des Verbrennungsmotors steigern (Abbildung 7). möglicht, Arbeitsprozesse an einer Baugruppe an unterschiedlichen Standorten durchzuführen, ohne dass sich Probleme mit den Datensätzen ergeben. Die Modulbauweise und das Baukastenprinzip

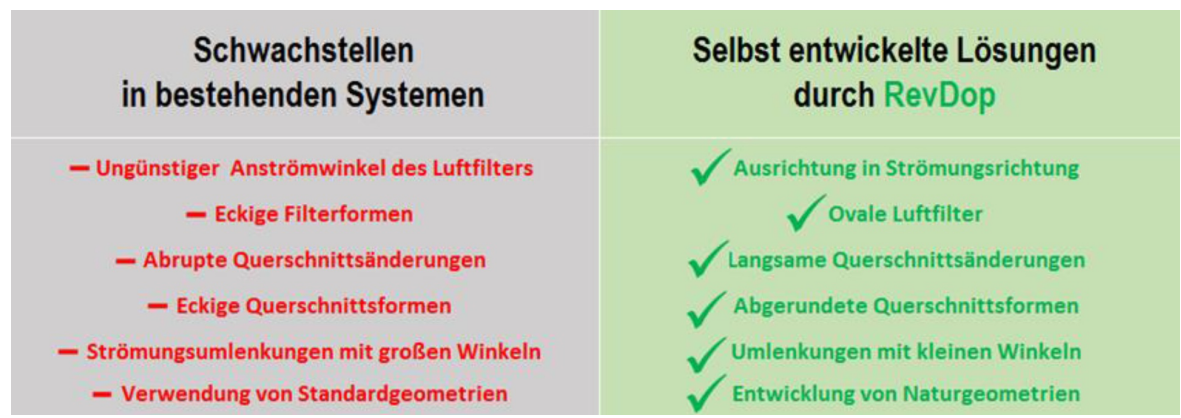

Abbildung 7: Schwachstellen bestehender Systeme (links), selbstentwickelte Lösungen durch RevDop

\subsubsection{Analyseverfahren zur Einschätzung des \\ Optimierungspotentials}

Zur wissenschaftlichen Evaluierung des Optimierungspotenzials, wurde ein Bewertungsschema entwickelt, welches eine Analyse aller Schwachstellen innerhalb eines Strömungssystems ermöglicht und eine Vergleichbarkeit der Ergebnisse im Hinblick auf den zu erwartenden Druckverlust garantiert. Hierfür wurden 6 Kriterien ausgearbeitet. Diese umfassen die Bewertung der Filterform, den Querschnittsformen, den Querschnittsänderungen, der Anströmung des Filterelements, der Bauteilverbindungen, und der Strömungsführung mit Umlenkungen.

\subsubsection{Parameterfreie}

\section{Konstruktion/Umsetzung von}

\section{Naturgeometrien}

Die Automobilkonzerne nutzen die parametrische Konstruktion, um Weltweit mehrere Konstrukteure in einem Netzwerk zu vereinen. Es wird somit er- von Volkswagen sind maßgeblich dafür. RevDop wendet hingegen die parameterfreie Modellierung an. Sie erfolgt spontan und beruht auf der Kreativität des Anwenders. Da RevDop naturorientierte, organische Strukturen entwickelt, ist die Auslegung der Strömungsbauteile nur parameterfrei umsetzbar. Dies setzt die freie, regellose Konstruktion voraus. Ebenfalls ist es durch diesen Konstruktionsablauf möglich, eine freie, kreative Modellierung umzusetzen, ohne an feste Konstruktionsregelwerke und Features gebunden zu sein. Um ideale, kompromisslose Bauteile entwickeln zu können, ist eine parameterfreie Modellierung deshalb unumgänglich.

\subsubsection{Langsame}

\section{Querschnittsänderungen zur}

\section{Vermeidung von Verwirbelungen}

Um optimale Ergebnisse bei der Druckverlustminimierung $\mathrm{zu}$ erreichen sind elementare Konstruktionspraxen anzuwenden. Um den Druckverlust eines Strömungsraumes mit zwei unterschied- 


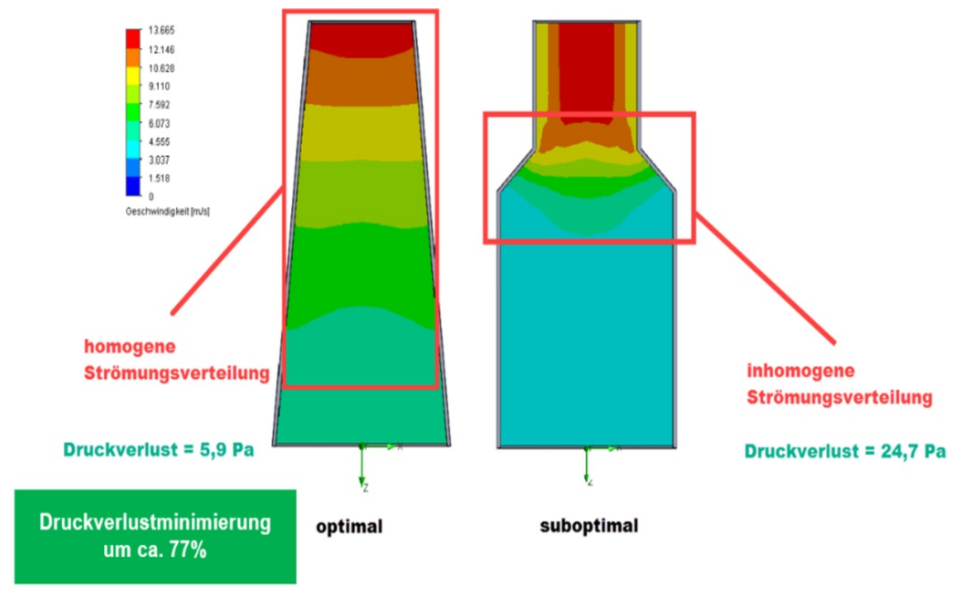

Abbildung 8: Vergleich abrupte, langsame Querschnittsänderungen

lich großen Querschnitten optimal zu verringern, wird eine langsame

Änderung mit flachen Winkeln angestrebt. So bilden sich weniger Strömungsverluste aus und der Gegendruck sinkt. Vermeidung von Kanten und starken Umlenkungen verbessern die Strömung maßgeblich (Abbildung 8).

\subsubsection{Strömungsführungen}

Ein weiteres Optimierungspotenzial ergibt sich bei Umlenkungen von Strömungskanälen. Im Gegensatz zu den häufig verwendeten Umlenkungen mit tels Standardrohrbögen in modernen Motoren, wird durch eine Veränderung des Strömungsraumes mittels parameterfreier Konstruktion der Druckverlust deutlich minimiert. Es kommt zeitgleich zu einer besseren Strömungsverteilung (Abbildung 9).

\subsubsection{Entwicklung eines}

\section{abgerundeten Filterelements}

Standard Filterelemente, wie sie in allen Serienfahrzeugen zu finden sind, werden als Plattenfilter bezeichnet. Es sind flache, eckige Filtereinsätze. Bei der

\section{Strömungsgeschwindigkeit bei einem Massenstrom von $0.18 \mathrm{~kg} / \mathrm{s}$}
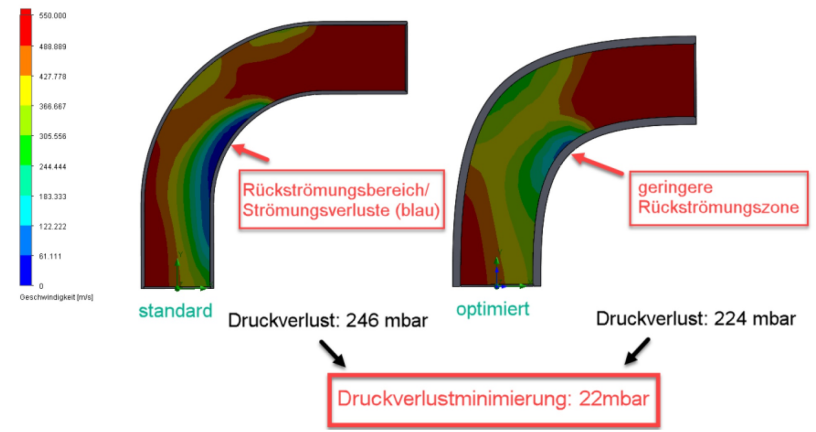

Abbildung 9: Vergleich eines Standardrohrbogens mit einem optimierten, naturorientierten Rohrbogen 
Durchströmung des Filters kommt es an den Eckbereichen zu Strömungsverlusten durch Verengung der Geometrie. RevDop entwickelt als erstes Unternehmen abgerundete Plattenfilter, um die Strömungsverluste im gesamten Ansaugsystem zu minimieren. Strömungssimulationen haben bewiesen, dass abgerundete Plattenfilter eine höhere Effizienz aufweisen. In Abbildung 10 werden die Unterschiede verdeutlicht.

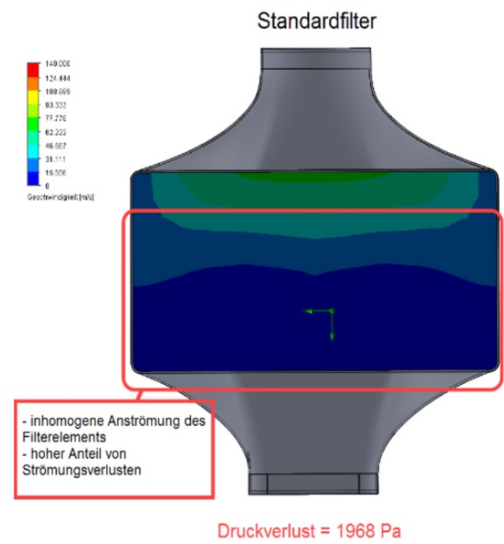

durch effizientere Ausnutzung des Querschnittes, bessere Filtereigenschaften der angesaugten Luft erreicht, wodurch die Langlebigkeit des Filters steigt. Folglich sinkt der Wartungsaufwand der Filterelemente, wodurch Kosten gespart und die Umwelt entlastet werden. Ebenfalls werden durch die bessere Filterung und durch die effizientere Ausnutzung des Filters der Kraftstoffverbrauch und der Schadstoffausstoß durchschnittlich



Abbildung 10: Vergleich Druckverlust Standardfilter und optimierter Filter

Der Standardfilter, mit den eckigen Randbereichen weist einen Bereich mit hohen Strömungsverlusten auf, hingegen werden bei dem optimierten Filter die Strömungsverluste minimiert. Zeitgleich werden durch die homogenere Durchströmung des optimierten Filterelements, gesenkt, da der Filter länger im optimalen Betriebszustand arbeitet.

\subsubsection{Optimale Anströmung des}

\section{Filterelementes}

Der Filter wird innerhalb des Ansaugsystems so angewinkelt, dass er optimal im

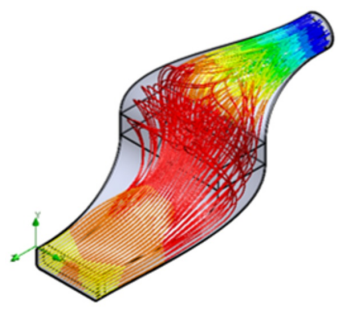

10,464

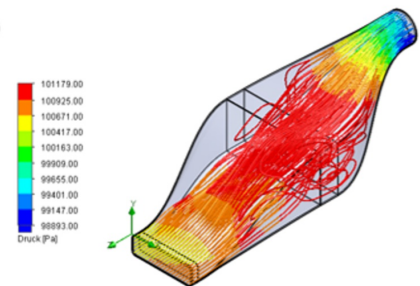

6,725

Abbildung 11: Vergleich Filteranströmung original/optimiert 
Strömungskanal liegt ohne in seiner Funktion beeinträchtigt zu werden (Abbildung 11). Durch diese günstig in Strömungsrichtung angewinkelte Konstruktion kann noch mehr potential genutzt und der Druckverlust gesenkt werden.

\subsubsection{Entwicklung} strömungsgünstiger

\section{Bauteilverbindungen}

Bei der Verbindung mehrerer Bauteile zu einem Strömungssystem ist es wichtig an den Übergängen eine homogene Strömungsverteilung zu gewährleisten.
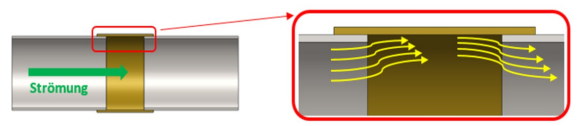

Abbildung 12: Schematische Darstellung von suboptimalen Bauteilübergängen

Diese suboptimale konstruktive Auslegung von Bauteilübergängen bei bestehenden Strömungssystemen moderner Fahrzeuge (Abbildung 12 und 13) ist verantwortlich für einen Teil der entstehenden Druckverluste im System.
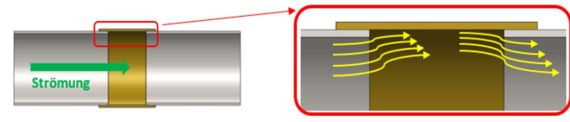

Abbildung 13: Schematische Darstellung optimierter Bauteilübergängen

Durch Weglassen solcher Verbindungsstellen werden diese Verluste vermieden. Eine weitere Möglichkeit bietet die Verwendung der RevDop-Verbindungsbauteile welche durch Analysen, Simulationen und Neukonstruktionen strömungsgünstig ausgelegt sind. Um das volle Potenzial zu nutzen, entwickelt RevDop eigene Verbindungsbauteile wie
Kompensatoren oder Konfusoren/Diffusoren und achtet hier besonders auf strömungsgünstige Lösungen die bei der Montage einfach zu handhaben sind. Dadurch werden an den Bauteilübergängen konstruktiv bedingte Rückströmungen, Verwirbelungen, Strömungsabrisse und Totwasserzonen verringert und/oder beseitigt (Abbildung 14). Dies wird durch Konstruktionen von glatten, fließenden, tangentialen Wandübergängen und Vermeidung von plötzlichen Querschnittsänderungen realisiert. Zur Darstellung des Optimierungspotentials suboptimaler Bauteilverbindungen bestehender Systeme wurde eine Strömungsanalyse zur Ermittlung des Druckverlustes durchgeführt (Abbildung 15). Durch diese konstruktive Geometrieänderung des Strömungskanals wird der Druckverlust um mehr als 75 Prozent gesenkt. Diese Auslegung ist auf weitere Bauteilverbindungen übertragbar.

\section{Reengineering als Service in der Produktentwicklung}

Aufgrund der hohen Herausforderungen in der hauseigenen Produktentwicklung hat das RevDop-Team ein breites Know How im Bereich der Produktentwicklung aufgebaut. Vor allem im Bereich der Automobilentwicklung bietet die RevDop GmbH Unterstützung in der Entwicklung physischer Produkte an. Dies betrifft die Rekonstruierung und Optimierung bestehender Bauteile aber auch die Neuentwicklung bis zum Status der Serienreife. Dabei setzt RevDop auf effiziente Prozesse und neueste Technologien, wie zum Beispiel die Topologieoptimierung, verschiedener 3D-Druckverfahren und zukünftig auch eine 3-dimensionale Präsentation des Entwicklungsstatus mithilfe von Virtual 
Reality. Ziel dabei sind schlanke Prozesse, bei der Realisierung individueller und innovativer Produktideen sowie eine schnelle Marktreife bei der Erfüllung hoher Qualitätsanforderungen. Die wichtigsten Serviceleistungen in der Produktentwicklung sind der mobile Messservice, das Reverse Engineering physischer Bauteile, die Umsetzung innovativer Produktideen durch eine individuelle Konstruktion, frühzeitige realistische Präsentationen der Entwicklungsphasen durch VR, Rendering und Rapid Prototiping, Strömungs- und Qualitätsanalysen. Strömungsbauteile können mithilfe von Topologieoptimierung in hohem Maße hinsichtlich der Minimierung des Druckverlustes optimiert werden.

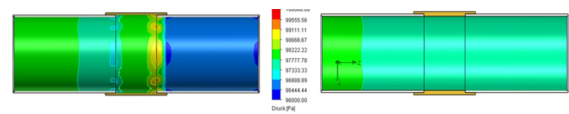

Abbildung 14: Analytische Ermittlung des entstehenden Druckverlustes

\subsection{Mobiler Messservice}

Zur Vermessung von Bauteilen wird ein Messarm der Marke Romer verwendet. Damit besteht die Möglichkeit Vermessungen direkt beim Kunden vor Ort vorzunehmen. Die Geometrien können dabei taktil und optisch aufgenommen werden. Dabei liegen Objektgrößen von $5 \mathrm{~mm}$ bis zu $10 \mathrm{~m}$ im Bereich einer realistischen Geometrievisualisierung. Der Messarm bietet eine hohe Flexibilität und je nach Toleranzanforderungen und Bauteilkomplexität kann vergleichsweise schnell vermessen werden. Es nahezu alle Materialien messbar. Auf einen Durchmesser von 2,5 m wird eine Wiederholgenauigkeit von $51 \mu \mathrm{m}$ sicher eingehalten. Zuverlässige Testergebnisse zeigen, dass üblicherweise 50 Prozent der
Wiederholgenauigkeit erreicht. Das Messgerät ist nach VDI/VDE 2617-9 Zertifiziert und hält damit den Anforderungen in der Automobilindustrie stand. Darüber hinaus ist der Messarm nach dem amerikanischen Standard B89.4.22 spezifiziert.

\subsection{Reverse Engineering}

Je nach Anforderung sind exakte (Nurbs) und parametrische Flächenrückführungen erforderlich. Beide Konstruktionsweisen kann RevDop abbilden. Wenn ein Bauteil für die Fertigung aus dem 3D-Drucker bestimmt ist, werden andere konstruktive Bedingungen an das CAD-Modell gestellt als an Modellen die für die konventionelle Fertigung gedacht sind. RevDop hat sich deshalb auch auf die Optimierung von CAD-Modellen für den 3D-Druck spezialisiert und erweitert dabei ständig das Portfolio an Konstruktionsrichtlinien, die sich bei den verschiedenen additiven Verfahren ergeben. Zudem können im Zuge des Reverse Engineering verschiedene Services, wie Erstbemusterungen, Wandstärkenanalysen Vermessung von Werkzeugen und digitale Bauteilreparaturen durchgeführt werden.

\subsection{Konstruktion}

Im Bereich der Konstruktion können Neukonstruktionen für innovative Produktideen aber auch Konstruktionsänderungen auf der Basis von Scanobjekten durchgeführt werden. Darüber hinaus kann hier mit der Erstellung von Strömungsräumen die Basis für eine Strömungsanalyse geschaffen werden.

\subsection{Rapid Prototyping}

In Kooperation mit dem Vinn:Lab der TH Wildau können bereits in sehr frühen Entwicklungsphasen Prototypen 
gefertigt werden. Dabei sind verschiedene Materialien vom thermoplastischen Kunststoff über Verbundwerkstoffe mit Glas- oder Carbonfasern umsetzbar. Verschiedene 3D-Drucker können dabei verschiedenen Anforderungen gerecht werden. Ob kleine Bauteile mit einem hohen Detailgrad oder große Bauteile. Es stehen Maschinen mit Bauräumen von bis 1 qm zur Verfügung. RevDop erarbeitet dabei momentan eine zuverlässige Vorgehensweise für die Nachbearbeitung von Oberflächen.

\subsection{Produktpräsentation}

Gute Projekte sollten professionell präsentiert werden. Hierfür nutzt RevDop das Rendering von Bauteilen. Zukünftig ist es geplant für eine reale Produktpräsentation die Virtual Reality Technologie in die Entwicklungsprozesse $\mathrm{zu}$ integrieren. Dadurch werden erforderliche Änderungen noch früher erkennbar, ohne dass ein physischer Prototyp erstellt werden muss. Dies kann helfen die Entwicklungsphase verkürzen und die Produktionskosten im Prototypenbau reduzieren. Darüber hinaus ergeben sich dadurch neue marketingpolitische Möglichkeiten in sehr frühen Entwicklungsstadien.

\subsection{Strömungs- und Qualitätsanalysen}

Es sind verschiedene Analyseszenarien denkbar. Vom Soll-/Ist-Vergleich über die Einhaltung von Form- und Lagetoleranzen, Verschleißanalysen und Strömungsanalysen von Flüssigkeiten und Gasen ist nahezu alles möglich. Eine Erhöhung der Energieeffizienz von Strömungssystemen ist hier, aufgrund der hauseigenen Produktentwicklung, als Spezialgebiet der RevDop GmbH Anzusehen.

\section{Literaturverzeichnis}

[BaSc15]

Dr.-Ing. E. h. van Basshuysen, Richard; Schäfer, Fred: Handbuch Verbrennungsmotor. Springer Vieweg, Wiesbaden, 2015.

[BüKa12]

Bühler, Karl; Kachel, Gerhard: „Topologieoptimierung für strömungsführende Bauteile im Fahrzeugentwicklungsprozess“ 2012, https:/ $/$ www.google.de/url?sa $=$ t\&rct $=j \& q=\& e s-$

$\mathrm{rc}=\mathrm{s} \&$ source $=$ web\&cd $=1$ \&ved $=0$ ahUKEwiArYy 4 0sTSAhXnC5oKHQSED_kQFggaMAA\&url=https $\% 3 \mathrm{~A} \% 2 \mathrm{~F} \% 2$ Fopus.hs-offenburg.de $\%$ 2Ffiles\%2F154\%2F2012_3.5_Buehler_Kachel_Topologieoptimierung.pdf\&usg $=\mathrm{AFQjCNE}$ _M6hdqIL8rcMHPEqUKOQlljHSg\&bvm= bv.148747831,d.bGs. Abruf am 2012-03-07

\section{[Fric12]}

Dr. Fricke, Holger: Topologieoptimierung Rohrbogen. 2012, http://www.ifam.fraunhofer.de/en/bremen/Klebetechnik_Oberflaechen/Klebetechnisch e_Fertigung/Fertigungstechnik/Fertigungsplanung/Simulation/Topologieoptimierung_Rohrbogen.html . Abruf am 2016-02-08

\section{[Gükd12]}

Gükduman, Hasan: Luftfiltersysteme, 2012, https://www.hg-motorsport.de/shop/in-

dex.php/cat/c18349_Airbox---Luftfiltersysteme.ht ml. Abruf am 2016-03-12

\section{[Hohe15]}

Hohe, Frederico: Audi Q3 Engine, 2015, http://www.carshowroom.com.au/reviews/audi-q320tdi-s-line-review-and-road-test/. Abruf am 2016-02-05.

\section{[KuBe09]}

Kuberczyk, Raffael; Berner, Hans-Jürgen; Bargende, Michael: Wirkungsgradunterschiede zwischen Otto- und Dieselmotor. Springer Fachmedien, MTZ Motortech, Wiesbaden, 2009. 
[Müll01]

Müller, Karl-Heinz: „Tuning-Tipps Teil1:

Grundlagen“, Waldkirchen, 2001

[Simo15]

Prof. Dr.-Ing. habil. Simon, Sylvio: Fachgespräche Druckverlustminimierung in Strömungssystemen von Verbrennungsmotoren, Interview, Brandenburgische Technische Universität Senftenberg-Cottbus, Senftenberg, 2015.

[Ston12]

Stone, Marvin: VW Catback Exhaust, 2012, http://www.goapr.co.uk/products/apr_exhaust_20 tfsi.html. Abruf am 2016-03-12

[Wimm12]

Univ.-Prof. Wimmer, Andreas: Thermodynamik des Verbrennungsmotors. Technische Universität Graz, Graz, 2012.

[Zell12]

Professor Dr.-Ing Zellbeck, Hans: Ladungswechselverluste effektiv reduzieren. 2012, http://vortraege.atzlive.de/Wissen/Dossiers/1328 /Ladungswechselverluste-effektiv-reduzieren.html. Abruf am 2016-02-05.

(C) $19 \begin{aligned} & \text { Dieser Beitrag ist unter der } \\ & \text { Creative-Commons-Lizenz }\end{aligned}$ 